\title{
Protein content of infant formula linked to obesity risk
}

Infant feeding methods have been linked to the risk of being overweight or obese later in life. Infants fed formula are more likely to become obese than breastfed infants, an observation that Koletzko et al. suggest is associated with the protein content of infant formula relative to that of breast milk.

Metabolic programming-factors such as metabolite or hormone concentrations during prenatal and postnatal development-affect health outcomes and risk of disease. Infant feeding practices might, therefore, alter childhood growth patterns. Previous studies have shown that high weight gain from birth to age 2 years is linked to a high prevalence of being overweight at school entry, and compared with infant formula feeding, breastfeeding can reduce the risk of obesity by school age by $15-25 \%$.
Koletzko and colleagues investigated whether the intake of protein is associated with growth patterns in infants from five European countries by randomly assigning them to receive one of two infant formulas with different protein contents. Infants given the formula with the higher protein content had notably higher body weight and BMI at the age of 2 years in comparison with those fed the lower protein formula.

The researchers postulate that high protein intake could lead to enhanced secretion of insulin and insulin-like growth factor I, which can boost growth during the first 2 years of life and increase adipogenic activity and adipocyte differentiation.

Lisa Richards

Original article Koletzko, B. et al. Can infant feeding choices modulate later obesity risk? Am. J. Clin. Nutr. 89 (Suppl.), 1502S-1508S (2009). 